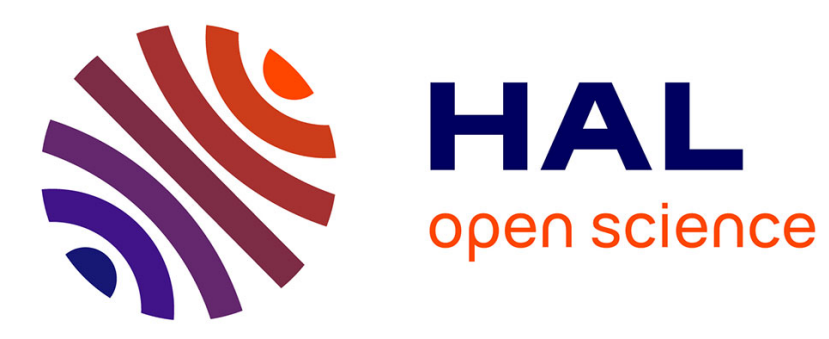

\title{
Identification of parametric models in the frequency-domain through the subspace framework under LMI constraints
}

Pauline Kergus, Fabrice Demourant, Charles Poussot-Vassal

\section{- To cite this version:}

Pauline Kergus, Fabrice Demourant, Charles Poussot-Vassal. Identification of parametric models in the frequency-domain through the subspace framework under LMI constraints. International Journal of Control, 2018, 10.1080/00207179.2018.1535717 . hal-02061484

\section{HAL Id: hal-02061484 \\ https://hal.science/hal-02061484}

Submitted on 3 Apr 2019

HAL is a multi-disciplinary open access archive for the deposit and dissemination of scientific research documents, whether they are published or not. The documents may come from teaching and research institutions in France or abroad, or from public or private research centers.
L'archive ouverte pluridisciplinaire HAL, est destinée au dépôt et à la diffusion de documents scientifiques de niveau recherche, publiés ou non, émanant des établissements d'enseignement et de recherche français ou étrangers, des laboratoires publics ou privés. 


\title{
Identification of parametric models in the frequency-domain through the subspace framework under LMI constraints
}

\author{
Pauline Kergus, Fabrice Demourant and Charles Poussot-Vassal *
}

\begin{abstract}
An extension of standard subspace-based algorithms to identify parametric systems from frequency response samples is presented. This algorithm uses arbitrary frequency spacing and allows frequency weighting. It offers the possibility to impose the model's poles location through LMI constraints. This technique is applied to a numerical example and to real industrial frequency-domain data originating from an openchannel flow for hydroelectricity production.
\end{abstract}

\section{INTRODUCTION}

Within system identification, experimental data of a system are used to characterize a model describing its dynamics, for simulation or control purposes. Considering that many systems can be characterized in the frequency domain, given samples of the frequency response of the system, determining a low order linear model is really useful for control design and analysis steps. In particular, subspace-based algorithms deliver estimated state-space models with no intermediate step either for SISO and MIMO systems indistinctly. Two frequency-domain subspace-based approaches are proposed in [1] and [2].

The subspace-based methods are well-established for Linear Time Invariant (LTI) systems for both time and frequency-domain. However, the obtained models are valid only for a single operating point. To tackle this issue, many methods have been developed in the area of Linear Parametrically Varying (LPV) modelling and identification, as detailed in [3]. In time-domain, subspace-based algorithms to identify LPV models with affine parameter dependence have been introduced in [4], [5] and [6] for example, relying on the reconstruction of the state sequence. As other global approaches in the area of LPV modelling, this method requires a unique experiment where the parameters entering the model vary in a known sequence. In this type of experiment, frequency-domain data are not relevant. Nevertheless, in many applications, as explained in [7], a parameter-dependent model is needed and must be identified on the basis of local experiments or simulations in which the parameter is frozen. This case, different from the traditional LPV framework, can be designated as parametric-LTI (pLTI), it allows to use frequency responses to characterize the system since the operating point remains unchanged during a local experiment.

The main contribution of this paper is to propose a subspace-based algorithm to identify a parametric LTI model from frequency-domain data. This stands as an extension of the original frequency-domain subspace-based method

${ }^{\star}$ ONERA, 2 avenue Edouard Belin, 31055 Toulouse, France

Contact: pauline.kergus@onera.fr presented in [1]. It preserves its two important features that are the frequency weighting capability, allowing to avoid sparse data in some regions of interests, and the ability to treat non uniformly spaced data, enabling to emphasize certain frequency ranges (see [1]). The formulation is close to the LTI identification scheme proposed in the two methods mentioned above. In this paper, we consider models with an affine parameter dependency, but the proposed method can be easily extended to polynomial dependency. Moreover, one of the main strength of our method is that it is possible to impose the poles location through LMI constraints for every considered operating point. This is also proposed in this article as an extension of what have been done in [8] and [9] in the LTI case only.

The outline of this paper is as follows. We start in Section II with the problem formulation and preliminary results on the identification of a LTI model through the frequencydomain subspace-based algorithm of [1]. In Section III, the extension of the classical frequency-domain subspace-based algorithm to identify p-LTI models is introduced and the possibility to use LMI constraints to impose poles location is detailed, both aspects constituting the core contribution. In Section IV, two numerical examples are presented. The first is purely academical while the second one is an industrial application representing an open-channel flow which behavior is described by partial differential equations. We end this paper with conclusions and discussion in Section V.

The following notations are used throughout the paper: the complex variable is denoted $\imath=\sqrt{-1}, \mathbb{R}$ and $\mathbb{C}$ designate respectively real and complex numbers. $\Re($.$) and \Im($.$) are$ respectively the real and imaginary parts of a complex number. $\mathbf{G}$ designates the considered system and $\mathbf{H}$ the identified model. $(.)^{T}$ is the matrix transposition and $\star$ is defined as follows:

$$
\left(\begin{array}{ll}
A & B \\
\star & C
\end{array}\right)=\left(\begin{array}{ll}
A & B \\
B & C
\end{array}\right) .
$$

\section{PRELIMINARY RESULTS}

\section{A. Problem formulation}

As mentioned in the introduction, subspace-based methods to identify a parametrized model of a system were developed in time-domain [7] [10] but not in the frequency one. Let us consider a parametric system $\mathbf{G}(\mathbf{p})$ with $n_{u}$ inputs and $n_{y}$ outputs where $\mathbf{p} \in \mathbb{R}^{n_{p}}$ is a vector of parameters that characterizes the operating point or the configuration ${ }^{1}$.

\footnotetext{
${ }^{1}$ Here, parameters can be the geometrical coefficients of a system, damping or mass values of a system for example.
} 
Given a set $\left\{\omega_{i}^{(j)}, \Phi_{i}^{(j)}\right\}\left(i=1 \ldots N, j=1 \ldots N_{p}\right)$, where $\Phi_{i}^{(j)} \in \mathbb{C}^{n_{y} \times n_{u}}$ represents the frequency response of $\mathbf{G}(\mathbf{p})$ obtained at pulsation $\omega_{i}^{(j)} \in \mathbb{R}_{+}$and at the operating point $\mathbf{p}^{(j)} \in \mathbb{R}^{n_{p}}$ and $N_{p}$ is the number of local samplings in the parameter; one can write:

$$
\Phi_{i}^{(j)}=\mathbf{G}\left(\mathbf{p}^{(j)}, \imath \omega_{i}^{(j)}\right) .
$$

The problem is to identify a linear model $\mathbf{H}(\mathbf{p})$ equipped with a $n^{\text {th }}$ order state-space realization as follows:

$$
\mathbf{H}(\mathbf{p}):\left\{\begin{array}{rl}
\mathbf{x}(t+1) & =A(\mathbf{p}) \mathbf{x}(t)+B(\mathbf{p}) \mathbf{u}(t) \\
\mathbf{y}(t) & =C(\mathbf{p}) \mathbf{x}(t)+D(\mathbf{p}) \mathbf{u}(t)
\end{array},\right.
$$

where $\mathbf{x}(t) \in \mathbb{R}^{n}$ is the state vector, $\mathbf{u}(t) \in \mathbb{R}^{n_{u}}$ the input vector of the system, $\mathbf{y}(t) \in \mathbb{R}^{n_{y}}$ the output vector, $A(\mathbf{p}) \in \mathbb{R}^{n \times n}, B(\mathbf{p}) \in \mathbb{R}^{n \times n_{u}}, C(\mathbf{p}) \in \mathbb{R}^{n_{y} \times n}$ and $D(\mathbf{p}) \in \mathbb{R}^{n_{y} \times n_{u}}$ and where all $A(\mathbf{p}), B(\mathbf{p}), C(\mathbf{p})$ and $D(\mathbf{p})$ matrices have an affine ${ }^{2}$ form as follows:

$$
M(\mathbf{p})=M_{0}+\sum_{k=1}^{n_{p}} \mathbf{p}_{k} M_{k},
$$

where $\mathbf{p}_{k}$ is the $k^{\text {th }}$ element of the vector $\mathbf{p}$. In this article, the proposed method is an extension of what have been proposed in [1], [8] and [9]. Since our procedure relies on the concatenation of all the LTI problems obtained at each operating point $\mathbf{p}^{(j)}$, the McKelvey algorithm (see [1]), which applies for LTI systems, is recalled in the next paragraph before detailing the proposed contribution in Section III.

\section{B. Preliminary results: the LTI case}

The algorithm presented in [1] is based on the extraction of a low-dimensional subspace by the use of a truncated singular value decomposition of a data matrix. The matrices of the state-space representation (4) are computed thanks to an estimation of the extended observability matrix (5). This method is recalled hereafter.

Let us consider a single operating point $\mathbf{p}^{(j)}$, the McKelvey algorithm allows finding a $n^{\text {th }}$ order model $\mathbf{H}_{j}$ of system $\mathbf{G}\left(\mathbf{p}^{(j)}\right)$ defined as:

$$
\mathbf{H}_{j}:\left\{\begin{array}{rl}
\mathbf{x}(t+1) & =A \mathbf{x}(t)+B \mathbf{u}(t) \\
\mathbf{y}(t) & =C \mathbf{x}(t)+D \mathbf{u}(t)
\end{array},\right.
$$

with $A \in \mathbb{R}^{n \times n}, B \in \mathbb{R}^{n \times n_{u}}, C \in \mathbb{R}^{n_{y} \times n}$ and $D \in \mathbb{R}^{n_{y} \times n_{u}}$.

To obtain this state-space representation, the extended observability matrix is defined as follows, where $q \geq n$ :

$$
\mathcal{O}_{q}=\left[\begin{array}{c}
C \\
C A \\
\vdots \\
C A^{q-1}
\end{array}\right] .
$$

Such a matrix is identified from samples of the frequency response $\left\{\omega_{i}^{(j)}, \Phi_{i}^{(j)}\right\}(i=1 \ldots N)$ obtained at the considered operating point $\mathbf{p}^{(j)}$. The following data matrices $G=$ $\frac{1}{N}\left[G_{1} \ldots G_{N}\right] \in \mathbb{C}^{q n_{y} \times n_{u} N}$ and $W=\frac{1}{N}\left[W_{1} \ldots W_{N}\right] \in$

\footnotetext{
${ }^{2}$ Affine forms are considered for simplicity, but polynomial extensions are straightforwardly derived.
}

$\mathbb{C}^{q n_{u} \times n_{u} N}$ are computed according to (6). For all $i=$ $1 \ldots N$, we have:

$$
\left\{\begin{aligned}
W_{i}^{T} & =\left[\begin{array}{lllll}
\Phi_{i}^{(j)} & e^{\imath \omega_{i}^{(j)}} \Phi_{i}^{(j)} & e^{\imath 2 \omega_{i}^{(j)}} \Phi_{i}^{(j)} & \ldots & e^{\imath(q-1) \omega_{i}^{(j)}} \Phi_{i}^{(j)}
\end{array}\right] \\
G_{i}^{T} & =\left[\begin{array}{llllll}
I_{n_{u}} & e^{\imath \omega_{i}^{(j)}} I_{n_{u}} & e^{\imath 2 \omega_{i}^{(j)}} I_{n_{u}} & \ldots & e^{\imath(q-1) \omega_{i}^{(j)}} I_{n_{u}}
\end{array}\right]
\end{aligned}\right.
$$

Then, a QR decomposition is applied in the first place:

$$
\left[\begin{array}{cc}
\Re(W) & \Im(W) \\
\Re(G) & \Im(G)
\end{array}\right]=\left[\begin{array}{cc}
R_{11} & 0 \\
R_{21} & R_{22}
\end{array}\right]\left[\begin{array}{c}
Q_{1}^{T} \\
Q_{2}^{T}
\end{array}\right],
$$

and the following rank revealing factorization (8) is performed on the matrix $M=R^{-1} R_{22}$ :

$$
M=\left[\begin{array}{ll}
\hat{U}_{s} & \hat{U}_{o}
\end{array}\right]\left[\begin{array}{cc}
\hat{\Sigma}_{s} & 0 \\
0 & \hat{\Sigma}_{o}
\end{array}\right]\left[\begin{array}{c}
\hat{V}_{s}^{T} \\
\hat{V}_{o}^{T}
\end{array}\right],
$$

where $R$ is a weighting matrix defined according to the assumptions on the noise (see [1] for details on how to build the weighting matrix). In the rest of the article, $R$ will be set to the identity matrix (no weighting is used). $\hat{\Sigma}_{s}$ contains the $n$ largest singular values. Then $n$ is the order of the estimated model and is fixed by the user. An extended observability matrix for a realization of $\mathbf{H}_{j}$ is then given by $\hat{\mathcal{O}}_{q}=\hat{U}_{s} \in \mathbb{R}^{q n_{y} \times n}$.

Granded on these definitions, the $A$ and $C$ matrices are estimated from this extended observability matrix $\hat{\mathcal{O}}_{q}$, through relation (9):

$$
\left(J_{1} R \hat{U}_{s}\right) A=J_{2} R \hat{U}_{s}, C=J_{3} R \hat{U}_{s},
$$

with:

$J_{1}=\left[\begin{array}{lll}I_{(q-1) n_{y}} & 0_{(q-1) n_{y} \times n_{y}}\end{array}\right], J_{2}=\left[\begin{array}{ll}0_{(q-1) n_{y} \times n_{y}} & I_{(q-1) n_{y}}\end{array}\right]$, $J_{3}=\left[\begin{array}{ll}I_{n_{y}} & 0_{n_{y} \times(q-1) n_{y}}\end{array}\right]$.

The remaining $B$ and $D$ matrices are the solutions of the following least square problem:

$\{B, D\}=\arg \min _{\tilde{B}, \tilde{D}} \sum_{i=1}^{N}\left\|\Phi_{i}^{(j)}-\tilde{D}-C\left(e^{\imath \omega_{i}^{(j)}} I-A\right)^{-1} \tilde{B}\right\|_{F}^{2}$

where $\|.\|_{F}$ denotes the Frobenius norm. Problem (11) can be solved in an efficient way using a linear solver.

\section{FREQUENCY-DOMAIN SUBSPACE-BASED ALGORITHM FOR THE IDENTIFICATION OF PARAMETRIC MODELS}

The subspace-based algorithm proposed in [1] allows to identify a model valid at one single operating point. Considering the structure of the matrices of the desired statespace representation (see equations (2) and (3)), we propose a way to concatenate all the LTI problems to solve the parametric problem directly.

\section{A. Proposed methodology}

Now that the LTI case has been recalled, we propose to consider all the local observability matrices $\hat{\mathcal{O}}_{q}^{(j)}$ computed as explained above for every operating point $\mathbf{p}^{(j)}$ where the data are available. Those are used to identify a parametric model $\mathbf{H}(\mathbf{p})$ as in equation (2). 
For the resolution of the parametric problem, the following notations are introduced:

$$
\Omega_{j}=J_{1} R \hat{\mathcal{O}}_{q}^{(j)}, \quad \Delta_{j}=J_{2} R \hat{\mathcal{O}}_{q}^{(j)}, \quad \Lambda_{j}=J_{3} R \hat{\mathcal{O}}_{q}^{(j)},
$$

where $J_{1}, J_{2}$ and $J_{3}$ are the same matrices as in the LTI case (see equation (10)). The matrix $R$ is still an eventual weighting function.

To be concatenated, these equations must be expressed in a unique basis, which is not guaranteed by the classical subspace-based algorithm of [1]. Since the McKelvey algorithm gives an extended observability matrix $\mathcal{O}_{q}^{(j)}$ for a representation of $\mathbf{G}\left(\mathbf{p}^{(j)}\right)$, it is possible to compute a transformation matrix $T_{j}$ for each available operating point $\mathbf{p}^{(j)}$ so that every LTI problem is expressed in the same basis corresponding to an observability canonical form. This form, which is unique, will be considered in this paper. As explained in [3], the computation of the transformation matrix $T_{j}$ consists in choosing $n$ independent rows of the full column rank observability matrix $\hat{\mathcal{O}}_{n}^{(j)} \in \mathbb{R}^{n_{y} n \times n}$. The selection of these $n$ independent rows is detailed in [3].

\section{B. Extension to the parametric case}

Finally, the $A(\mathbf{p})$ and $C(\mathbf{p})$ matrices of the parametric model satisfy:

$$
A\left(\mathbf{p}^{(j)}\right)=T_{j} A^{(j)} T_{j}^{-1}, \quad C\left(\mathbf{p}^{(j)}\right)=C^{(j)} T_{j}^{-1},
$$

where $A^{(j)}$ and $C^{(j)}$ are the solution of Equation (9) of the LTI identification problem at the operating point $\mathbf{p}^{(j)}$.

Considering Equations (2) and (9), and the transformation into the observable canonical form (13), finding the $A(\mathbf{p})$ and $C(\mathbf{p})$ matrices of the parametric model consists in solving the following sets of equations $\forall j=1 \ldots N_{p}$ :

$$
\Omega_{j} T_{j} A\left(\mathbf{p}^{(j)}\right) T_{j}^{-1}=\Delta_{j}, C\left(\mathbf{p}^{(j)}\right) T_{j}^{-1}=\Lambda_{j},
$$

where the matrices $\Omega_{j}, \Delta_{j}, \Lambda_{j}$ are obtained as in (12). Then, following equations (3) and (14), the matrices $A_{k}, k=$ $0 \ldots n_{p}$ and $C_{k}, k=0 \ldots n_{p}$ forming $A(\mathbf{p})$ and $C(\mathbf{p})$ respectively are computed as follows:

$$
\Psi A_{a u g}=\Delta_{a u g}, \Gamma C_{a u g}=\Lambda_{a u g}
$$

with $\Psi \in \mathbb{R}^{N_{p}(q-1) n_{y} \times n_{p} n}, A_{a u g} \in \mathbb{R}^{n_{p} n \times n}$ and $\Delta_{a u g} \in$ $\mathbb{R}^{N_{p}(q-1) n_{y} \times n}$ defined by:

$$
\begin{aligned}
& \Psi=\left[\begin{array}{cccc}
\Omega_{1} T_{1} & \mathbf{p}_{1}^{(1)} \Omega_{1} T_{1} & \cdots & \mathbf{p}_{n_{p}}^{(1)} \Omega_{1} T_{1} \\
\vdots & \vdots & & \vdots \\
\Omega_{N_{p}} T_{N_{p}} & \mathbf{p}_{1}^{\left(N_{p}\right)} \Omega_{N_{p}} T_{N_{p}} & \cdots & \mathbf{p}_{n_{p}}^{\left(N_{p}\right)} \Omega_{N_{p}} T_{N_{p}}
\end{array}\right], \\
& A_{\text {aug }}^{T}=\left[A_{0} A_{1} \ldots A_{n_{p}}\right], \Delta_{\text {aug }}^{T}=\left[\Delta_{1} T_{1} \ldots \Delta_{N_{p}} T_{N_{p}}\right] \text {. }
\end{aligned}
$$

and with $\Gamma \in \mathbb{R}^{N_{p} n_{y} \times n_{p} n_{y}}, C_{a u g} \in \mathbb{R}^{n_{p} n_{y} \times n}$ and $\Lambda_{a u g} \in$ $\mathbb{R}^{N_{p} n_{y} \times n}$ :

$$
\Gamma=\left[\begin{array}{cccc}
I_{n_{y}} & \mathbf{p}_{1}^{(1)} I_{n_{y}} & \ldots & \mathbf{p}_{n_{p}}^{(1)} I_{n_{y}} \\
\vdots & \vdots & & \vdots \\
I_{n_{y}} & \mathbf{p}_{1}^{\left(N_{p}\right)} I_{n_{y}} & \ldots & \mathbf{p}_{n_{p}}^{\left(N_{p}\right)} I_{n_{y}}
\end{array}\right]
$$

$C_{a u g}^{T}=\left[C_{0} C_{1} \ldots C_{n_{p}}\right], \Lambda_{a u g}^{T}=\left[\Lambda_{1} T_{1} \ldots \Lambda_{N_{p}} T_{N_{p}}\right]$

Finally, the $B_{k}, D_{k}$ matrices, for $k=1 \ldots n_{p}$, forming $B(\mathbf{p})$ and $D(\mathbf{p})$ are the solutions of the following least square problem:

$$
\begin{aligned}
\min \sum_{j=1}^{N_{p}} \sum_{i=1}^{N} & \| \Phi_{i}^{(j)}-D\left(\mathbf{p}^{(j)}\right)-C\left(\mathbf{p}^{(j)}\right) \\
& \left(e^{j \omega_{i}^{(j)}} I-A\left(\mathbf{p}^{(j)}\right)\right)^{-1} B\left(\mathbf{p}^{(j)}\right) \|_{F}^{2} .
\end{aligned}
$$

The whole procedure to identify a parametric model as formulated in (2) is summed up in Algorithm 1.

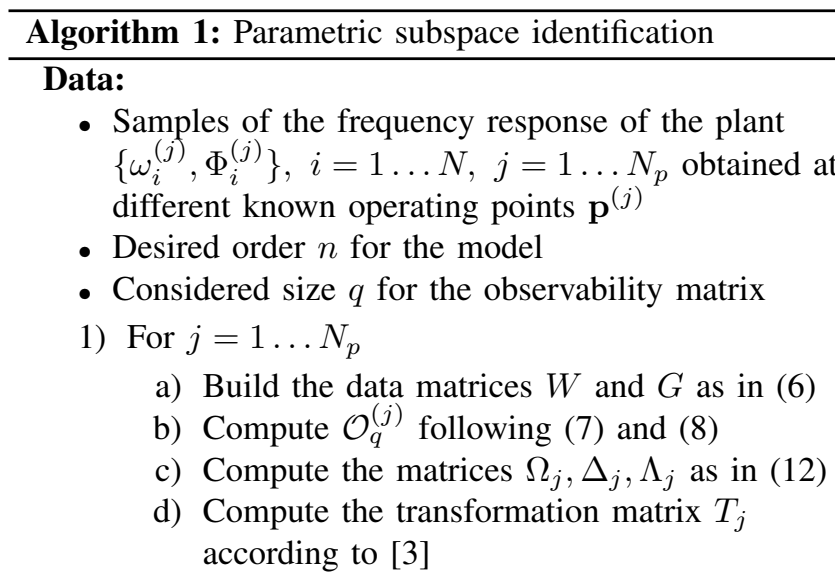

2) Build the matrices $\Psi, \Delta_{a u g}, \Gamma$ and $\Lambda_{a u g}$ according to (15) and get the matrices $A(\mathbf{p})$ and $C(\mathbf{p})$ of the parametric model given in (2)

3) Solve the least square problem given in (16) to get the matrices $B(\mathbf{p})$ and $D(\mathbf{p})$

Remark 1: In practice, if one does not want the matrices $B$ and $D$ to be parameter-dependant, the key is to adapt the least square problem given in Equation (16), in this case, $B(\mathbf{p})=B_{0}$ and/or $D(\mathbf{p})=D_{0}$.

Remark 2: The extension of Algorithm 1 to have the parameters enter the model in a polynomial way is straightforward, the matrices $\Psi$ and $\Gamma$ from Equation (15) have to be adapted. More specifically, a column should be added for each polynomial term appearing in the structure of the state-space representation matrices. However, the affine form offers interesting properties in terms of convexity that allows to impose poles location through LMI constraints as explained in the next paragraph. Last but not least, it should be noted that the parameter dependency needs to be a priori determined so that the matrices $\Psi$ and $\Gamma$ can be computed according to the corresponding structure. If one needs a hint on the parameter dependence, it is possible to use the Loewner interpolation technique for p-LTI systems proposed in [11] as a complement.

Remark 3: Note that the frequency weighting proposed in [1] that appears during the computation of the matrices $\Omega_{j}, \Delta_{j}, \Lambda_{j}$ (see Equation (12)) through the matrix $R$ can be different according to the considered operating point. 


\section{LMI regions constraints}

As a second contribution, the above parametric model computation can be performed under LMI constraints to impose the poles location, as explained in this paragraph.

In [8] and [9], a modified frequency-domain subspace method allowing to introduce constraints on the identified model poles location is presented. This formulation is based on an LMI description of regions in continuous/discrete map (where the stability domain is a particular case). Here, we propose to adapt this LMI approach to impose the poles location of the identified parametric model of Equation (2).

First, let us recall some preliminary results on LMI regions which were presented in [12] and [13]. A LMI region $\mathcal{D}$ is defined through two matrices $P=P^{T} \in \mathbb{R}^{r \times r}$ and $Q \in$ $\mathbb{R}^{r \times r}$ :

$$
\mathcal{D}=\left\{z \in \mathbb{C} \mid P+Q z+Q^{T} \bar{z}<0\right\} .
$$

By extension of what has been done in [8] and [9] in the LTI case, the eigenvalues of the matrix $A(\mathbf{p})$ lie in $\mathcal{D}$ if and only if $X=X^{T}>0 \in \mathbb{R}^{n \times n}$ exists such that the following LMI is verified:

$$
\forall \mathbf{p} \in \Delta, P \otimes X+Q \otimes(A(\mathbf{p}) X)+Q^{T} \otimes(A(\mathbf{p}) X)^{T}<0,
$$

where $\Delta=\left\{\mathbf{p} \mid \forall k=1 \ldots n_{p}, \mathbf{p}(k) \in[0,1]\right\}$ is a convex set (we assume that the parameters are normalized). Its convex hull is defined by $\Delta_{0}=\left\{\mathbf{p} \mid \forall k=1 \ldots n_{p}, \mathbf{p}(k) \in\{0,1\}\right\}$ For a fixed $\mathbf{x} \in \mathbb{R}^{r n}$, we define the function $f_{\mathbf{x}}: \Delta \rightarrow \mathbb{R}$ by:

$$
f_{\mathbf{x}}(\mathbf{p})=\mathbf{x}^{T}\left(P \otimes X+Q \otimes(A(\mathbf{p}) X)+Q^{T} \otimes(A(\mathbf{p}) X)^{T}\right) \mathbf{x} .
$$

Since $A(\mathbf{p})$ is an affine function of $\mathbf{p}$, the function $f_{\mathbf{x}}(\mathbf{p})$ is a convex function of $\mathbf{p}$. We then have the following property (see [14] for details):

$$
f_{\mathbf{x}}(\mathbf{p})<0 \forall \mathbf{p} \in \Delta \Longleftrightarrow f_{\mathbf{x}}(\mathbf{p})<0 \forall \mathbf{p} \in \Delta_{0} .
$$

Obviously this means that, to ensure that the eigenvalues of $A(\mathbf{p})$ lies in $\mathcal{D}$, one just needs to check the LMI given in Equation (18) for $\mathbf{p} \in \Delta_{0}$, which contains a finite number of elements.

By denoting $\tilde{A}(\mathbf{p})=A(\mathbf{p}) X=\tilde{A}_{0}+\sum_{k=1}^{n_{p}} \mathbf{p}(k) \tilde{A}_{k}$ with $\tilde{A}_{k}=A_{k} X, k=1 \ldots n_{p}$, finding the matrices $A_{k}$ forming $A(\mathbf{p})$ ensuring that the poles of the system lie in $\mathcal{D}$ is done by solving the following problem, $\beta>0$ :

$$
\min _{\tilde{A}_{k}, X, \beta>0} \beta
$$

subject to:

$$
\begin{gathered}
P \otimes X+Q \otimes \tilde{A}(\mathbf{p})+Q^{T} \otimes \tilde{A}(\mathbf{p})^{T}<0, \forall \mathbf{p} \in \Delta_{0} \\
\left(\begin{array}{cc}
I & \left(\Omega_{j} T_{j} \tilde{A}\left(\mathbf{p}^{(j)}\right)-\Delta_{j} T_{j} X\right) \\
(\star)^{T} &
\end{array}\right)>0 \\
X=X^{T}>0 .
\end{gathered}
$$

According to the definition of the convex hull $\Delta_{0}$, Equation (22) gives a set of $2^{n_{p}}$ LMIs to be verified. Equation (23) comes from the fact that one wants to minimize $\left\|\Omega_{j} T_{j} A\left(\mathbf{p}^{(j)}\right)-\Delta_{j} T_{j}\right\|_{2}, \forall j=1 \ldots N_{p}$ (see Equation (14)) to fit the data.
The procedure to identify a parametric model with constraints on the poles location is summed up in the following Algorithm 2.

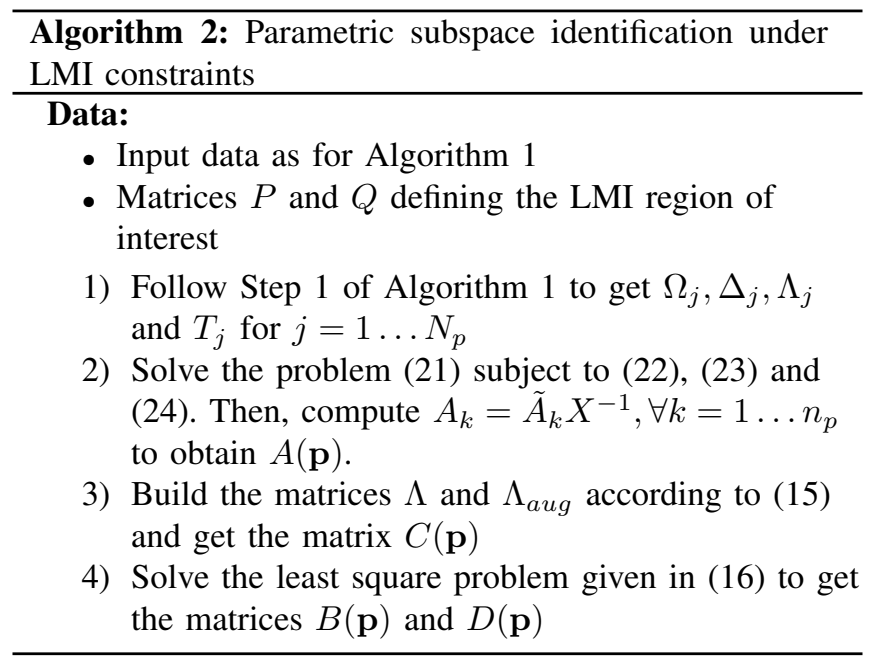

Remark 4: It should be noted that the consistency of the problem is not affected by the use of LMIs if the constraints are relevant with the system to identify (see [1], [9]).

Remark 5: In most cases, one wants the dynamical process to be stable, so the poles of $A(\mathbf{p})$ must lie in the unit circle corresponding to the LMI region $\mathcal{D}$ defined by:

$$
P=\left(\begin{array}{cc}
-1 & 0 \\
0 & -1
\end{array}\right), Q=\left(\begin{array}{ll}
0 & 0 \\
1 & 0
\end{array}\right) .
$$

Additional LMI regions examples can be found in [9].

Remark 6: Note that Equation (20) is valid only if the function $f_{\mathbf{x}}(\mathbf{p})$ defined in (19) is convex, which is the case when the desired model has an affine parameter dependency. If it is not the case, it is still possible to impose the location of the poles for the different considered operating points $\mathbf{p}^{(j)}$ by using the classical LMIs defined in [8] and [9]. This is frequently done in local LPV approaches even if there is no guarantee on the poles location between the considered operating points $\mathbf{p}^{(j)}$.

\section{NUMERICAL APPLICATIONS}

In order to illustrate the main features of the algorithm presented above, simulation examples are presented. The first one is a simple academical one while the second one is obtained from an industrial simulation of an open channel flow described by partial differential equations.

\section{A. Academical example}

Here, we consider a second order SISO system with varying damping factor and static gain according to a scheduling parameter $p \in[0,1]$. The objective is to identify a parametric model on the basis of two frequency responses obtained at two different operating point, $p^{(1)}=0.2$ and $p^{(2)}=0.8$ ( $n_{p}=1$ and $N_{p}=2$ ). The system is the following one:

$$
\mathbf{G}(p, z)=\frac{1+p}{z^{2}+0.01 p z+0.99},
$$


and the sampling period is $T_{s}=1 \mathrm{~ms}$. According to (2) and (25), one wants to identify a second order LPV model ( $n=2$ ) of the following form:

$$
\mathbf{H}(p):\left\{\begin{aligned}
\mathbf{x}(t+1) & =\left(A_{0}+p A_{1}\right) \mathbf{x}(t)+\left(B_{0}+p B_{1}\right) \mathbf{u}(t) \\
\mathbf{y}(t) & =\left(C_{0}+p C_{1}\right) \mathbf{x}(t)+\left(D_{0}+p D_{1}\right) \mathbf{u}(t)
\end{aligned}\right.
$$

We used $N=400 \operatorname{logspaced~samples~of~the~two~frequency~}$ responses of $\mathbf{G}\left(p^{(1)}\right)$ and $\mathbf{G}\left(p^{(2)}\right)$ between 0.1 rad.s $s^{-1}$ and the Nyquist pulsation $\frac{\pi}{T_{s}}$ rad.s $s^{-1}$. No frequency weighting is used in this example. The results of the identification are given in Figure 1 for the noise-free case.

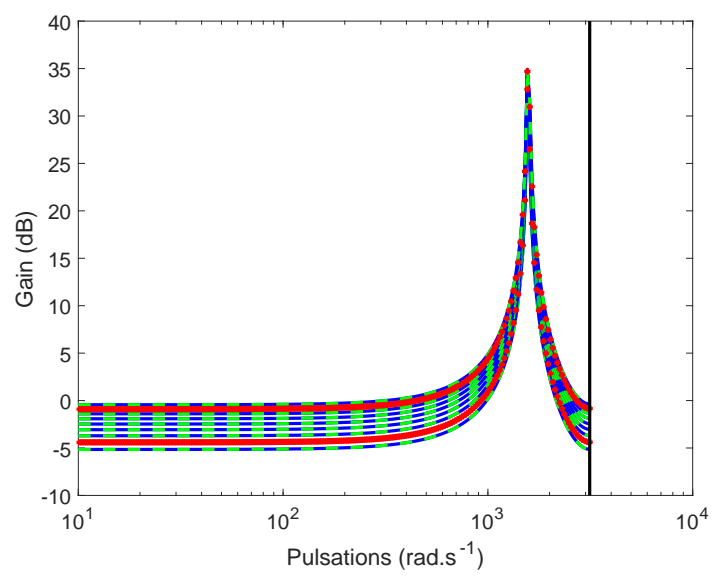

Fig. 1. Identification of the parametric model $\mathbf{H}(s, p)$ on the basis of noise-free data: the blue solid lines correspond to frequency responses of the obtained $\mathrm{p}$-LTI model for $p=0.1 k, k=1 \ldots 9$, the red points to the frequency responses of the local systems $\mathbf{G}\left(p^{(1)}\right)$ and $\mathbf{G}\left(p^{(2)}\right)$ from which the data for the identification come from, and the dashed green lines are the frequency responses of the real transfers $\mathbf{G}\left(p^{(k)}\right), p^{(k)}=0.1 k, k=1 \ldots 9$

Figure 1 shows that our algorithm successfully identified a parametric model that shows a coherent behavior between the different values of the operating point. Obviously, in this example, we knew that the system has an affine dependence in $p$, so the proposed model is consistent with the problem considered here. The matrices of the discrete state-space representation are given in Equation (27), note that the expected observable canonical form is recovered.

$$
\begin{array}{cc}
A(p)=\left[\begin{array}{cc}
0 & 1 \\
-0.99 & 0.01 p
\end{array}\right], B(p)=\left[\begin{array}{c}
0 \\
1+p
\end{array}\right], \\
C(p)=\left[\begin{array}{cc}
1 & 0
\end{array}\right], & D(p)=0,
\end{array}
$$

Since $\mathbf{G}(p, z)$ is stable, using Algorithm 1 or 2 gives the same result in the noise-free case. However, when considering noisy data, the identified model is unstable for some values of the parameter $p$. Noise is added as follows, with a signal to noise ratio $S N R=5$ :

$$
\forall i, j, \Phi_{i}^{(j)}=\mathbf{G}\left(p^{(j)} \imath \omega_{i}\right)\left(1+N_{i}\right),
$$

where $N_{i}=10^{-\frac{S N R}{10}}(\operatorname{randn}(1)+\imath \operatorname{randn}(1))$. Using Algorithm 1 with this noisy dataset leads to an unstable system for some values of $p$, see Figure 2. It is then interesting to use Algorithm 2 with a stability constraint since the system is known to be stable. This is a common problem when identifying a parametric model on the basis of noisy experimental data (see [15]).

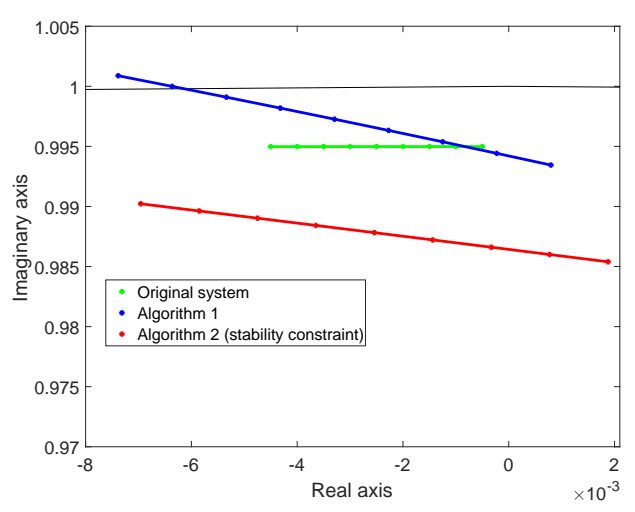

Fig. 2. Poles of the parametric model identified using Algorithm 1 (blue points) or Algorithm 2 with a stability constraint (red points) for $p=0.1 k, k=1 \ldots 9$. The unit circle (limit of stability) is indicated in black, the poles of the original system are in green. There are two complex conjugate poles, only the evolution of one pole is represented here.

Figure 2 illustrates the fact that Algorithm 2 allows to constraint the poles location for all $p \in\left[\begin{array}{ll}0 & 1\end{array}\right]$ for an affine parameter dependence, which is particularly useful when working with noisy data.

\section{B. Application to an open channel flow for hydroelectricty}

Now let us move to an industrial problem provided by the French power producer EDF (Electricité de France). EDF uses water resources to generate green energy with run-of-the-river power plants. The behavior relies on openchannel hydraulic systems that are non-linear and which dynamic depends on the operating point. Their physical model requires partial differential equations (namely SaintVenant equations). In [16], a new irrational transfer function is proposed for open channels to represent the level-to-flow variations for any operating point. It is the solution of SaintVenant equations under many assumptions. The system has two inputs, the entering and the outgoing flows $q_{e}$ and $q_{s}$, and one output, the water depth $h$. The transfer is given by:

$$
G\left(x, s, Q_{0}\right)=G_{e}\left(x, s, Q_{0}\right) q_{e}(s)+G_{s}\left(x, s, Q_{0}\right) q_{s}(s)
$$

where

$$
\begin{aligned}
& G_{e}\left(x, s, Q_{0}\right)=\frac{\lambda_{1}(s) e^{\lambda_{2}(s) L+\lambda_{1}(s) x}-\lambda_{2}(s) e^{\lambda_{1}(s) L+\lambda_{2}(s) x}}{B_{0} s\left(e^{\lambda_{1}(s) L}-e^{\lambda_{2}(s) L}\right)} \\
& G_{s}\left(x, s, Q_{0}\right)=\frac{\lambda_{1}(s) e^{\lambda_{1}(s) x}-\lambda_{2}(s) e^{\lambda_{2}(s) x}}{B_{0} s\left(e^{\lambda_{1}(s) L}-e^{\lambda_{2}(s) L}\right)}
\end{aligned}
$$

where $x$ is the position of the measurement point on the channel, $Q_{0}$ the nominal flow, $L$ the length of the open channel. $B_{0}, \lambda_{1}(s)$ and $\lambda_{2}(s)$ depend on the canal configuration and the nominal flow (see [16] for details). The system, which dynamic is visible on Figure 3, is extremely slow, has a delay behavior and a pole in limit of stability. Moreover, it has an infinite number of poles since the transfer function is irrational. Due to this complexity, a low-order and parameter dependent model well tailored is needed to quickly simulate the behaviour of a channel and to design a controller. In this paper, we propose to identify such a parametric model for a varying measurement point $x$ (allowing them to simulate the 
process at varying measurement points). Note that a model has been identified in [16] through the Loewner framework but for a varying discharge $Q_{0}$.

In this application, the delays ( $\tau_{e}$ and $\tau_{s}$ for the entering and outgoing flows $q_{e}$ and $q_{s}$ respectively) are known, they depend on the measurement point $x$ and the canal configuration:

$$
\tau_{e}=\frac{x}{c_{0}+V_{0}}, \tau_{s}=\frac{L-x}{c_{0}-V_{0}} .
$$

As it has been done in [16], we will identify a model $\hat{H}$ of the "delay-free" system $H$ defined by the following equation $\left(Q_{0}=1400 \mathrm{~m}^{3} \cdot \mathrm{s}^{-1}\right.$ is fixed):

$$
\begin{aligned}
& H(x, s)=\left[G_{e}\left(x, s, Q_{0}\right) e^{+\tau_{e} s} G_{s}\left(x, s, Q_{0}\right) e^{+\tau_{s} s}\right] \\
& \hat{H}(x, s)=\left[R_{e}(x, s) R_{s}(x, s)\right] \\
& \hat{G}(x, s)=\left[R_{e}(x, s) e^{-\tau_{e} s} R_{s}(x, s) e^{-\tau_{s} s}\right]
\end{aligned}
$$

The model $\hat{G}$ of the actual system is then obtained by adding the delays to the previously identified "delay-free" model $\hat{H}$. The samples of the frequency response of the system without considering the delays $\left\{\omega_{i}^{(j)}, \Phi_{i}^{(j)}\right\}, i=$ $1 \ldots N, j=1 \ldots N_{p}$ are estimated directly through its irrational transfer for five different measurement points $x^{(j)} \in$ $\{100,3925,7750,11575,15400\} m\left(N_{p}=5\right)$ :

$$
\forall j=1 \ldots N_{p}, \forall i=1 \ldots N, \Phi_{i}^{(j)}=H\left(x_{j}, \imath \omega_{i}^{(j)}\right)
$$

The same frequencies are used for every operating point: 250 pulsations uniformly spaced between $10^{-4}$ rad.s $s^{-1}$ and $5.10^{-2} \mathrm{rad} . \mathrm{s}^{-1}$. The results of the identification of the delayfree system are given on Figure 3. It shows that our method successfully identified a model of order $n=10$ of the delay-free system with an affine dependence of $x$. This application is really interesting: from a partial differential equation of great complexity, our method allows to obtain a parametric model which is simple to evaluate and to simulate for different measurement points.

In this case, the assumption of affine parameter dependence is probably limitating but still shows good results.

\section{CONCLUSIONS}

In this paper, an algorithm to identify parametric systems with an affine (or polynomial) parameter dependence through the subspace framework has been proposed. The formulation is close to the LTI identification scheme proposed in [1] and [2], it uses frequency-domain data obtained at different operating points. For an affine parameter dependence, the set of parameters is convex and our algorithm offers the possibility to impose the poles location through LMI constraints as it has been done in [8] and [9] for the LTI case. The method was illustrated on a numerical example and has shown good results on an industrial application consisting in an openchannel for hydroelectricty. So far, we consider parametric models where the parameter is frozen. An interesting outlook for this work would be to take into account the variation dynamics of the parameters, for example by by including their dynamics into the state-space representation.
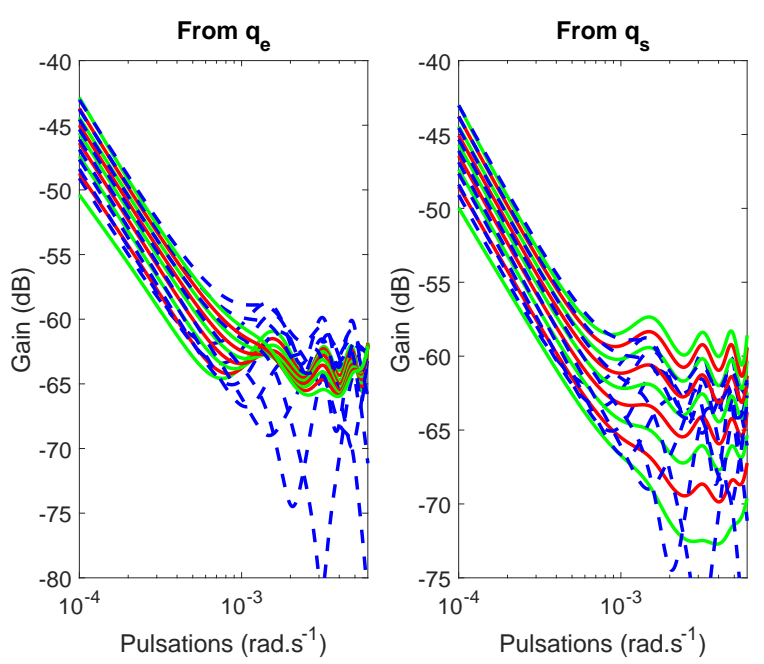

Fig. 3. Identification of the delay free system $\tilde{\mathbf{G}}$ : the $10^{\text {th }}$ order model $\hat{\mathbf{H}}(x)$ is in solid green for the 5 measurement points $x^{(j)} \in$ $\{100,3925,7750,11575,15400\} m$ and in solid red for other values $x \in$ $\{2013,5837,9663,13487\} \mathrm{m}$. The dashed blue lines are the transfers of the delay free system $\tilde{\mathbf{G}}$ estimated from the irrational transfer function for the different points $x$ used above.

\section{REFERENCES}

[1] T McKelvey, H Akçay, and L Ljung. Subspace-based multivariable system identification from frequency response data. Transactions on Automatic Control, 1996.

[2] K Liu, R Jacques, and D Miller. Frequency domain structural system identification by observability range space extraction. Journal of dynamic systems, measurement and control, 1996.

[3] R Tóth. Modeling and identification of linear parameter-varying systems. 2010.

[4] JW Van Wingerden and M Verhaegen. Subspace identification of multivariable LPV systems: A novel approach. In International Conference on Computer-Aided Control Systems, 2008.

[5] P Gebraad, JW Van Wingerden, G Van Der Veen, and M Verhaegen. Lpv subspace identification using a novel nuclear norm regularization method. In American Control Conference, 2011.

[6] V Verdult and M Verhaegen. Subspace identification of multivariable linear parameter-varying systems. Automatica, 2002.

[7] M Lovera and G Mercere. Identification for gain-scheduling: a balanced subspace approach. In American Control Conference, 2007.

[8] D Miller and R De Callafon. Subspace identification with eigenvalue constraints. Automatica, 2013.

[9] F. Demourant and C. Poussot-Vassal. A new frequency-domain subspace algorithm with restricted poles location through lmi regions and its application to a wind tunnel test. International Journal of Control, 2016.

[10] V Verdult. Non linear system identification: a state-space approach. 2002

[11] AC Ionita and AC Antoulas. Data-driven parametrized model reduction in the loewner framework. SIAM Journal on Scientific Computing, 2014

[12] M Chilali, P Gahinet, and P Apkarian. Robust pole placement in $1 \mathrm{~m}$ regions. Transactions on Automatic Control, 1999.

[13] M Chilali and P Gahinet. $H_{\infty}$ design with pole placement constraints: an LMI approach. Transactions on Automatic Control, 1996.

[14] C Scherer and S Weiland. Linear matrix inequalities in control. Lecture Notes, Dutch Institute for Systems and Control, Delft, 2000.

[15] C. Poussot-Vassal, F. Demourant, A. Lepage, and D. Le Bihan. Gust load alleviation: a sub/transonic wind tunnel experimental validation of a 2d aeroelastic airfoil. Transactions on Control Systems Technology, 2017.

[16] V. Dalmas, G. Robert, C. Poussot-Vassal, I. Pontes Duff, and C. Seren. From infinite dimensional modelling to parametric reduced order approximation: Application to open-channel flow for hydroelectricity. In 15th European Control Conference, 2016. 